\title{
Diagnosis of PLMD from increased pulse rate variability on overnight oximetry
}

\author{
This article was published in the following Dove Press journal: \\ Nature and Science of Sleep \\ 13 July 2010 \\ Number of times this article has been viewed
}

\section{Uma M Krishnaswamy \\ Sean E Higgins \\ Christopher A Kosky \\ Simone deLacy \\ Adrian J Williams}

Sleep Disorders Unit, Guys' and St. Thomas' NHS Trust, London, England, UK
Correspondence: Uma M Krishnaswamy Sleep Disorders Unit, St. Thomas' Hospital, Lambeth Palace Road, London SEI 7EH, England, UK

Tel +44207l883446

Fax +44 20 7II 86115

Email uma_eshwari@hotmail.com
Study objective: This study was undertaken in a group of patients with periodic limb movement disorder (PLMD) to assess whether the presence of increased pulse rate variability (PRV) without desaturation on overnight oximetry was suggestive of the occurrence of periodic limb movements (PLMs).

Methods: Seventy sleepy patients with a polysomnographic diagnosis of PLMD and 25 controls with obstructive sleep apnea were included in this retrospective study. All patients had undergone initial domiciliary oximetry and subsequent polysomnography (PSG). The oximetry tracings were independently interpreted by five sleep unit personnel for the presence of increased PRV. Further, the association between increased PRV and PLMs was evaluated in the summary graph of the PSG.

Results: Fifty seven (81.4\%) patients had definite evidence of increased PRV without episodes of desaturation on initial oximetry, which was later confirmed to be due to PLMs on PSG. $13(18.6 \%)$ patients had no PRV on oximetry and PSG but had PLMD. The inter-interpreter concurrence in suspecting a diagnosis of PLMD based on oximetry alone was more than $80 \%$ in $64(91 \%)$ patients.

Conclusion: The presence of isolated increased PRV on overnight oximetry is a valuable tool in suspecting nonsleep apnea disorders like PLMD.

Keywords: periodic limb movements, pulse rate variability, oximetry

\section{Introduction}

Overnight oximetry is a commonly used case-finding tool in patients with suspected sleep apnea in the UK. In the latter disorder, the oximetry trace typically shows repetitive desaturation associated with fluctuations in pulse rate.

Although sleep apnea is one of the commonest causes of excessive daytime sleepiness, there are many other causes for sleepiness, such as poor sleep hygiene, narcolepsy, idiopathic hypersomnolence, circadian rhythm disorders, restless legs syndrome with/without periodic limb movements (PLMs), and certain drugs. Moreover, many of these disorders can occur in conjunction with sleep apnea.

One of the sleep disorders in which repeated arousals result in fragmented sleep is periodic limb movement disorder (PLMD). PLMs can occur as part of restless legs syndrome (RLS) or as an isolated phenomenon. PLMs cause sympathetic activation, which result in fluctuations in heart rate and blood pressure during sleep. The latter may account for the apparent association of PLMs with cardiovascular and cerebrovascular disease. ${ }^{1-3}$ 
The occurrence of these fluctuations in pulse rate has not been used to identify PLMs in patients screened with overnight oximetry to date. The present study was undertaken in a group of patients with polysomnographically proven PLMD to assess whether the presence of increased pulse rate variability (PRV) in the overnight oximetry trace was indicative of the occurrence of PLMs.

\section{Background}

In an earlier study undertaken in our unit, ${ }^{4}$ spectral analysis of the heart rate trace on PSG was performed in 10 normal subjects and 47 patients with various sleep disorders. It was seen that there were distinct changes in the heart rate in patients with obstructive sleep apnea (OSA), non-obstructive hypoventilation, and PLMD. The changes in heart rate in patients with PLMD occurred regardless of the presence of EEG arousals. Thus, we postulated that the presence of heart rate variability in the absence of desaturation may be an indicator of non-sleep apnea disorders like PLMD.

In a subsequent pilot study, ${ }^{5} 6$ sleepy patients with isolated increased PRV in the oximetry trace were identified. These patients underwent nocturnal polysomnography (nPSG) to look for the underlying sleep disorder as well as the cause for these variations in pulse rate. It was found that all these patients had PLMD.

The present study was undertaken to further demonstrate that isolated PRV on overnight oximetry may indicate the occurrence of PLMs. As interpretation of the oximetry trace was visual, the degree of concurrence amongst 5 independent interpreters in identifying fluctuations in pulse rate suggesting the occurrence of PLMs was also analyzed.

\section{Materials and methods}

This retrospective study was conducted in the Sleep Disorders Unit of a university hospital in London. The study group included consecutive patients with a polysomnographic diagnosis of PLMD who had undergone prior overnight oximetry followed by PSG over a 7-year period. The control group consisted of patients with OSA, as the latter also causes PRV.

Overnight oximetry graphs of the study and control groups were given to 5 interpreters ( 3 physicians and 2 senior sleep technologists) for independent analysis. Each oximetry study was printed on A4 paper using a scale of $70 \%-100 \%$ for oxygen saturation $\left(\mathrm{SaO}_{2}\right)$ and $30-120$ beats per minute for pulse. The timescale used was the complete recording (usually $6-8$ hours). The scorers did not use the electronic record on the computer screen as the number of data points exceeds the number of pixels on the screen. Hence the average data displayed by the software which gave an appearance of smoothed signals was used.

A 19-inch screen with a graphics card resolution of $1280 \times 1024$ was used; this meant that each data point displayed on the screen represented an average of 25 samples. To eliminate this 'data averaging', it was necessary to view the record in a 20-minute timescale. Since the analytical method relied on pattern recognition, printed records were used.

The summary graph of the whole night's recording derived as described above was printed in 2 formats. The first was a conventional graph available as part of the overnight report (Figure 1A), and the second with a minimum - maximum trace derived from the software in order to visualize PRV in greater detail (Figure 1B).

All interpreters were blinded with respect to the history and polysomnographic diagnosis. They were required to record their observations on the oximetry traces provided in a predesigned form (Appendix 1) and to give a global impression based on visual analysis as to whether the patient had one of the following: normal oximetry, OSA, PLMD, or OSA with PLMD.

Prior to interpretation, the scorers underwent a 45 minute group standard-setting session where they were shown examples of PRV and standard criteria for recognizing PRV, and oxygen desaturations were established. If a variation in the pulse rate was associated with desaturation (defined as a $4 \%$ drop or more in arterial $\mathrm{SaO}_{2}$ from baseline), then the arousal was due to a respiratory event. If the variation was not associated with desaturation, it was defined as 'isolated PRV'. Thus, oximetry could show: (a) oxygen desaturation associated with PRV; (b) episodic, isolated PRV; (c) continuous, isolated PRV; and (d) normal saturation and pulse trace.

After interpretation of the overnight oximetry, concurrence amongst the interpreters with respect to the following was computed: (a) presence/absence of PRV, and (b) prediction of the occurrence of PLMs based on the oximetry.

\section{Explanation for 'increased PRV' by visual analysis}

Traditionally, demonstrating increased heart rate variability has been by computer analysis of the R-R interval. This increased heart rate during or after an arousal can also be appreciated visually on an epoch of PSG (Figure 2A). 


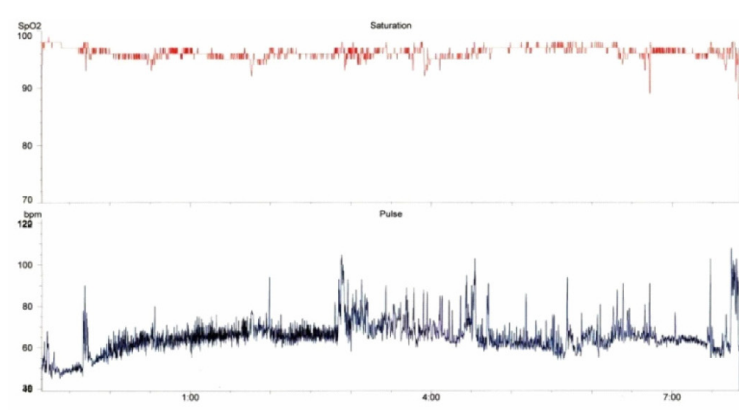

A

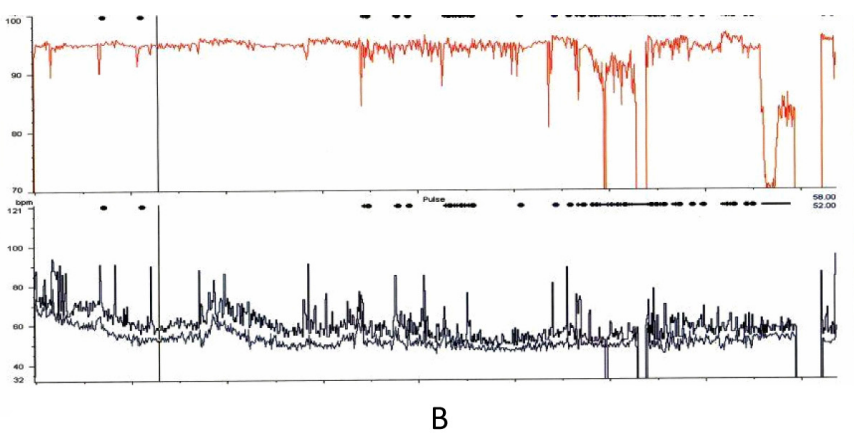

B

Figure I The 2 formats of oximetry traces used for interpretation. A) Conventional trace. B) Trace with minimum-maximum format of pulse waveform.

Overnight oximetry includes oxygen saturation and a pulse trace in compressed format. Hence the arousals appear as an increased 'density' of the pulse rate trace on oximetry (Figure 2B). Furthermore, the baseline PRV for an individual (related to sinus arrhythmia) can be judged from the very beginning of the recording, ie, presumed wake leading on to presumed sleep. If there is an increase in the density and amplitude of the pulse rate pattern, this can reasonably be assumed to represent increased heart rate variability.

\section{Statistical analysis}

All computations were done using the statistical applications available in Microsoft Excel. Descriptive statistics were used

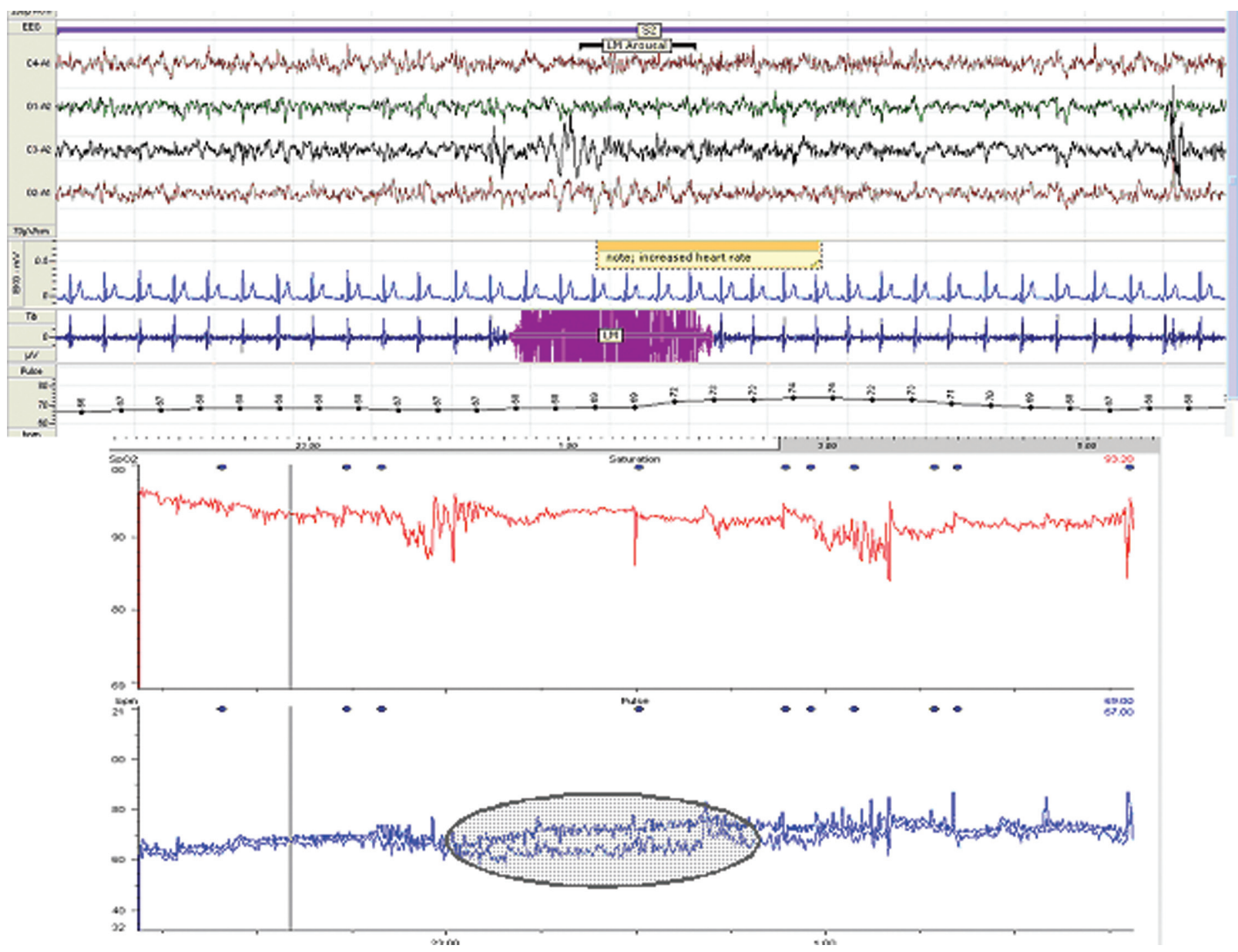

Figure 2 Demonstration of heart/pulse rate variability related to arousal. A) An epoch of polysomnography. B) Overnight oximetry (shaded area indicates pulse rate variability related to arousal). 
for patient demography and clinical profile. Concurrence was computed as the percentage of interpreters reporting the same findings on oximetry.

\section{Results}

\section{Demography and clinical features}

174 patients had a diagnosis of PLMD in the study period (2000-2007). 87 (50\%) patients had undergone a home oximetry prior to the PSG, of whom only 70 patients were included in the study, as the remaining 17 patients had a poor quality oximetry trace. Oximetry traces of 25 patients with varying severity of OSA were included as the control group.

The demographic and clinical features of all PLMD patients as well as the 2 subgroups (with and without PRV on oximetry) are detailed in Table 1.

$35(50 \%)$ patients had preexisting mild to moderate sleep apnea and were persistently sleepy despite optimal CPAP (continuous positive airway pressure) therapy. Hence, oximetry followed by PSG (as the former was reportedly normal except for fluctuations in pulse rate) had been performed to look for an additional sleep disorder.

Co-morbid illnesses included epilepsy in 6 (8.5\%), diabetes in $2(2.9 \%)$, chronic renal failure and peripheral neuropathy in 1 patient each. None of the patients included in the study were on beta blockers, calcium channel blockers, tricyclic antidepressants, or dopaminergic agonists, all of which are known to modify the heart rate and/or affect autonomic response.

Table I Demographic and clinical profile of PLMD patients

\begin{tabular}{|c|c|c|c|}
\hline Parameter & $\begin{array}{l}\text { All } \\
\text { patients } \\
(n=70)\end{array}$ & $\begin{array}{l}\text { Patients } \\
\text { with PRV } \\
(n=57)\end{array}$ & $\begin{array}{l}\text { Patients } \\
\text { without } \\
\text { PRV } \\
(n=13)\end{array}$ \\
\hline Male:Female & $52: 18$ & 44: 13 & $8: 5$ \\
\hline Age (mean $\pm S D)$ & $48.6 \pm 14$ & $48.4 \pm 13.9$ & $50.8 \pm 14$ \\
\hline \multicolumn{4}{|l|}{ Presenting symptoms } \\
\hline Excessive daytime sleepiness & 49 (70\%) & $4 \mathrm{I}(7 \mathrm{I} .9 \%)$ & $12(92.3 \%)$ \\
\hline Restless legs & 30 (42.8\%) & $22(38.6 \%)$ & $8(61.5 \%)$ \\
\hline Nocturnal limb movements & $32(45.7 \%)$ & $25(43.8 \%)$ & $7(53.8 \%)$ \\
\hline Fatigue & $14(20 \%)$ & $11(19.3 \%)$ & $3(23 \%)$ \\
\hline Insomnia & $5(7 \%)$ & $4(7 \%)$ & I (7.7\%) \\
\hline \multicolumn{4}{|l|}{ Co-morbid illnesses } \\
\hline Diabetes & $2(2.8 \%)$ & $2(3.5 \%)$ & 0 \\
\hline Epilepsy & $6(8.5 \%)$ & $5(8.8 \%)$ & I (7.7\%) \\
\hline Renal failure & I (I.4\%) & 0 & I (7.7\%) \\
\hline Peripheral neuropathy & I (I.4\%) & 0 & I (7.7\%) \\
\hline Post cardiac transplant & $\mathrm{I}(\mathrm{I} .4 \%)$ & 0 & I (7.7\%) \\
\hline
\end{tabular}

Abbreviations: PLMD, periodic limb movement disorder; PRV, pulse rate variability.

\section{Oximetry interpretation in study and control groups}

All the interpreters were able to discriminate between the OSA and the non-OSA group of patients based on the occurrence of desaturation with PRV in the former and isolated or no PRV in the latter group. The findings on analysis of the PLMD group alone are presented.

\section{PRV in patients with PLMD}

On inspection of the oximetry trace alone, 57 (82.4\%) patients had evidence of isolated PRV. Among these patients, 38 (66.6\%) had PRV throughout the night and 19 (33.3\%) had episodic PRV.

\section{Inter-interpreter concurrence in suspecting PLMD}

On compiling the results of the oximetry interpretation by the 5 sleep unit personnel, there was $>80 \%$ inter-interpreter concurrence in suspecting the presence of PLMs based on the occurrence of increased PRV on overnight oximetry in $64(91 \%)$ patients (Table 2$)$.

\section{PRV in the summary graph of PSG}

After computing the concurrence amongst interpreters, the summary graph of the PSG was visually analyzed by another independent observer to look for the occurrence of PRV in relation to PLMs (Figure 3 and Figure 4). It was seen that PLMs were associated with PRV in all those patients who had been identified as 'suspected PLMD' based on oximetry alone. Further, those patients who had not had PRV in the home oximetry $(n=13)$ had no PRV in relation to PLMs in the PSG as well. The severity of PLMD in the PSG is depicted in Table 3.

\section{Discussion}

This study illustrates the significance of increased PRV in oximetry studies that would otherwise be reported as normal because of the absence of desaturation. The presence of

Table 2 Inter-observer concurrence in suspecting PLMD based on oximetry

\begin{tabular}{ll}
\hline Concurrence (\%) & Number of patients (\%) \\
\hline$>80 \%$ & $64(91 \%)$ \\
$60 \%-80 \%$ & $6(9 \%)$ \\
$<60 \%$ & 0
\end{tabular}

Abbreviation: PLMD, periodic limb movement disorder. 


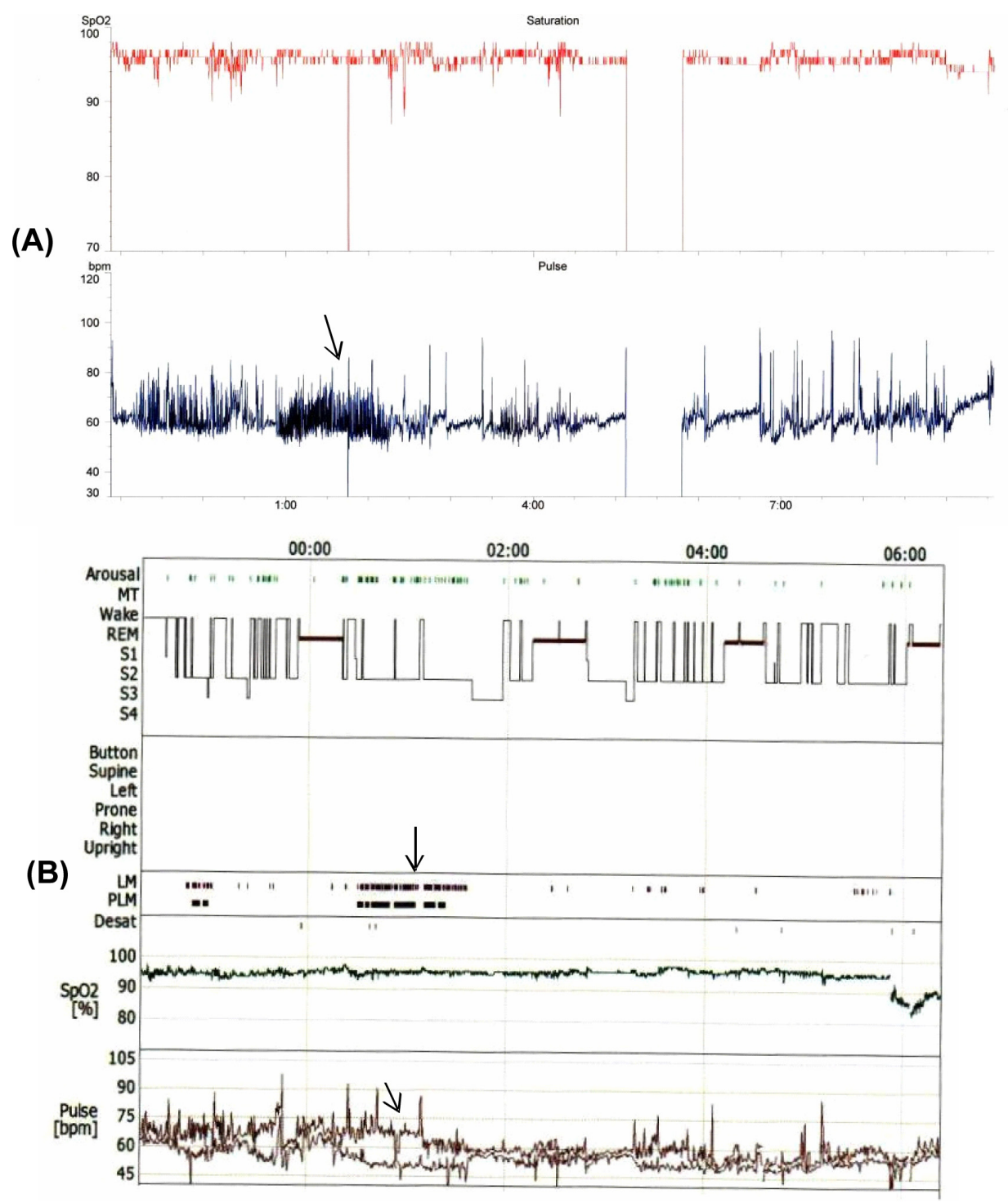

Figure 3 Oximetry trace (A) and summary graph of the polysomnography (B) in a patient with mild periodic limb movement disorder. Arrows denote increased pulse rate variability on oximetry and a similar pattern associated with periodic limb movements on polysomnography.

isolated fluctuations in pulse rate during sleep is indicative of a change in sleep stage or the occurrence of autonomic activation. The latter can occur with arousals due to any cause (spontaneous, respiratory event, or limb movement related) and cardiac arrhythmias.

Table 3 Severity of PLMD on PSG

\begin{tabular}{ll}
\hline Severity (PLMI) & Number of patients $(\mathbf{n}=\mathbf{7 0})$ \\
\hline Mild $(5-25)$ & $34(47.1 \%)$ \\
Moderate $(25-50)$ & $19(27.1 \%)$ \\
Severe $(>50)$ & $13(18.5 \%)$ \\
No PSG (actigraphy) & $4(5.7 \%)$ \\
Total & 70 \\
\hline
\end{tabular}

Abbreviations: PLMD, periodic limb movement disorder; PSG, polysomnography PLMI, periodic limb movement index.
PLMs have been shown to be associated with sympathetic activation which results in autonomic arousals. In the literature published to date, ${ }^{6-9}$ the occurrence of sympathetic activation during PLMs has been used to prove an association between RLS/PLMD and the occurrence of cardiovascular disease. In these studies, sympathetic activation was measured by spectral analysis of heart rate variability on the ECG and changes in blood pressure.

The novelty in our study lies in the fact that the presence of isolated increased PRV on overnight oximetry has thus far not been used as an indicator of the occurrence of PLMs. Hence the utility of oximetry outside the setting of sleep apnea is limited. Although standard oximetry software can analyze the degree of PRV and produce indices 
(A)

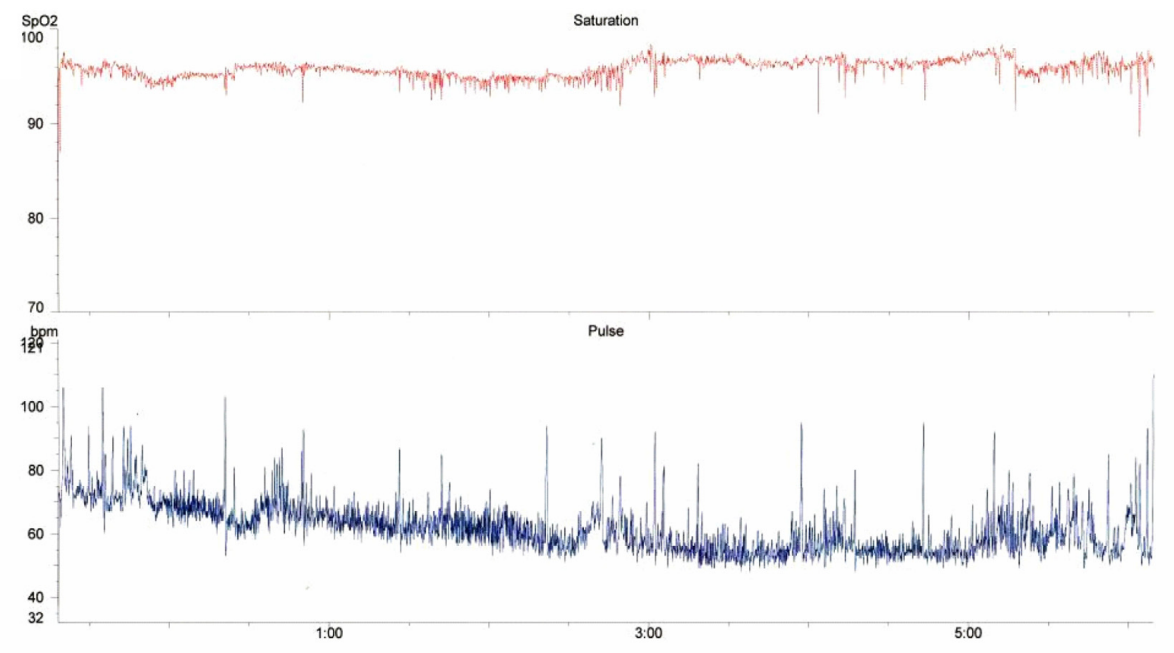

(B)

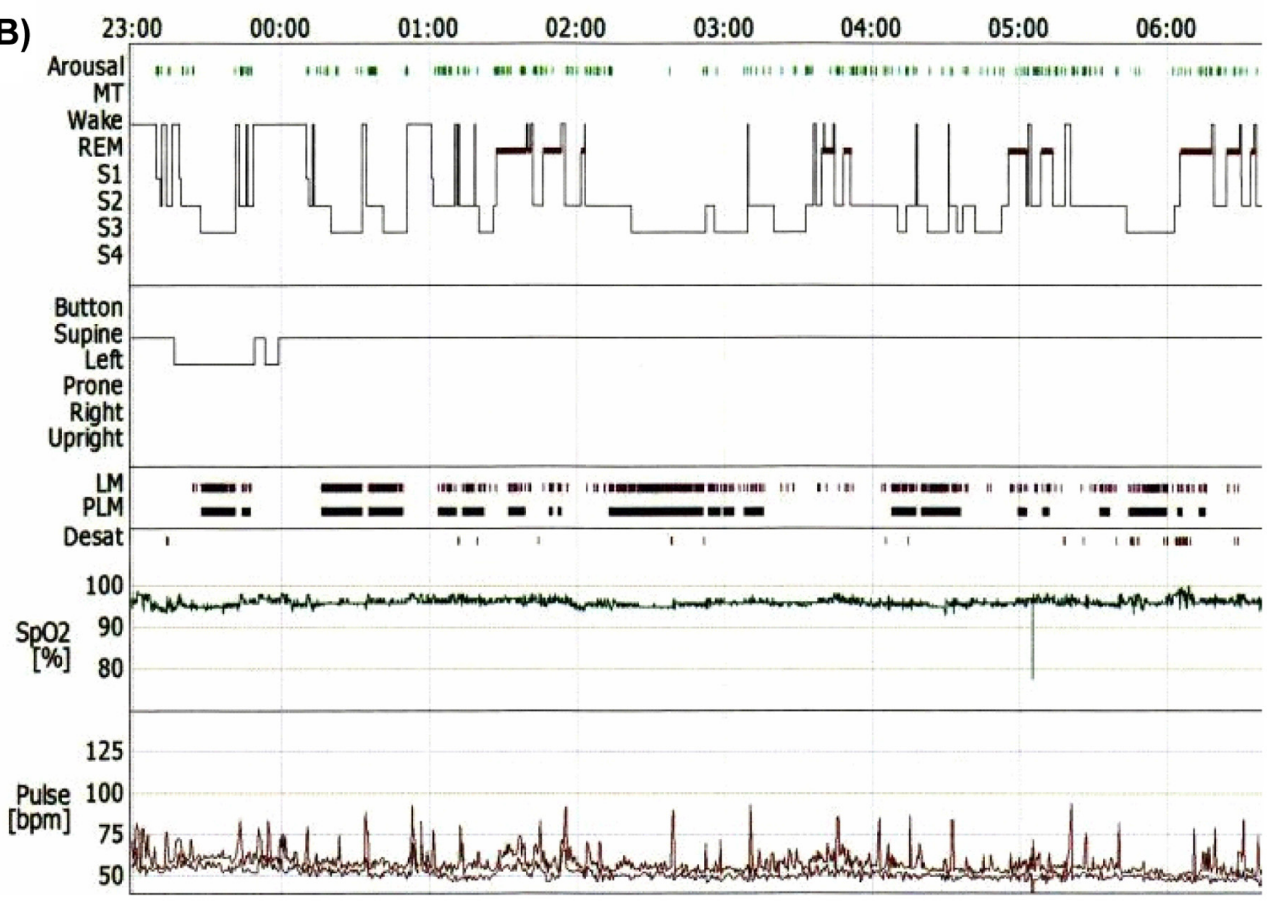

Figure 4 Oximetry trace (A) and summary graph of polysomnography $(\mathbf{B})$ in a patient with severe periodic limb movement disorder. Pulse rate variability seen throughout the night on oximetry and a similar pattern coinciding with periodic limb movements on polysomnography.

such as the number of 6 beat/min rises per hour, there is no published data on normal values for PRV. In this study, we have demonstrated that visual analysis of the overnight oximetry trace may indicate the occurrence of PLMs if the presence of PRV is identified. In addition, there was more than $80 \%$ inter-observer concurrence in suspecting PLMD based on visual analysis of the oximetry trace alone, which also correlated with the occurrence of PLMs on PSG.

One of the drawbacks of the study was its retrospective nature. Although only patients with PLMD were included, the interpreters were blinded with respect to the polysomnographic findings and the final diagnosis. In addition, there was a control group of patients with OSA.
A prospective study in which all sleepy patients with isolated PRV on overnight oximetry tracings are subjected to PSG would be useful to further define the pattern and occurrence of PRV in other non-apnea sleep disorders as well, thus diversifying the utility of overnight oximetry.

\section{Conclusion}

In conclusion, the occurrence of isolated, increased PRV in overnight oximetry tracings is an indicator of the occurrence of PLMs in sleep.

\section{Disclosure}

The authors report no conflicts of interest in this work. 


\section{References}

1. Winkelman JW, Shahar E, Sharief I, Gottlieb DJ. Association of restless legs syndrome and cardiovascular disease in the Sleep Heart Health Study. Neurology. 2008;70:35-42.

2. Walters AS, Rye DB. Review of the relationship of restless legs syndrome and periodic limb movements in sleep to hypertension, heart disease and stroke. Sleep. 2009;32(5):589-597.

3. Walters AS, Rye DB. Evidence continues to mount on the relationship of restless legs syndrome/periodic limb movements in sleep to hypertension, cardiovascular disease, and stroke. Sleep. 2010;33(3): 287.

4. Nooman YM, Hamid S, Higgins SE, Howard RS, Williams AJ. A study of heart rate variability in sleep related breathing disorders. Am J Resp Crit Care Med. 1999;159:A4222.
5. Higgins S, Bonakis B, Merritt S, De Lacy S, Kosky C, Williams AJ. Can assessment by pulse oximetry be useful outside the setting of sleep disordered breathing; Glasgow, Scotland: ESRS Sleep Congress; 2008.

6. Sforza E, Nicolas A, Lavigne G, Gosselin A, Petit D, Montplaisir J. EEG and cardiac activation during periodic leg movements in sleep: support for a hierarchy of arousal responses. Neurology. 1999;52:786.

7. Winkelman JW. The evoked heart rate response to periodic leg movements of sleep. Sleep. 1999;22:575-580.

8. Sforza E, Pichotb V, Barthelemyb JC, Haba-Rubioa J, Rocheb F. Cardiovascular variability during periodic leg movements: a spectral analysis approach. Clin Neurophysiol. 2005;116:1096-1104.

9. Siddiqui F, Strus J, Ming X, Lee IA, Chokroverty S, Walters AS. Rise of blood pressure with periodic limb movements in sleep and wakefulness. Clin Neurophysiol. 2007;118:1923-1930. 


\section{Appendix I}

Appendix I Sample response sheet for interpretation of overnight oximetry

\begin{tabular}{lll}
\hline & Question & Patient ID (O I, O2...) \\
\hline I & PRV & Yes/No \\
2 & Pattern of PRV & Episodic/Throughout the night \\
3 & $\begin{array}{l}\text { Is PRV associated with } \\
\text { desaturation? }\end{array}$ & Yes/No \\
4 & Overall impression & OSA/Normal/PLMD/OSA and PLMD \\
5 & Comments & \\
\hline
\end{tabular}

Note: If response to Question I is 'Yes', answer questions 2-5.

Abbreviations: PRV, pulse rate variability; OSA, obstructive sleep apnea; PLMD, periodic limb movement disorder.

\section{Publish your work in this journal}

Nature and Science of Sleep is an international, peer-reviewed, open access journal covering all aspects of sleep science and sleep medicine, including the neurophysiology and functions of sleep, the genetics of sleep, sleep and society, biological rhythms, dreaming, sleep disorders and therapy, and strategies to optimize healthy sleep. The journal welcomes

original research, clinical \& epidemiological studies, reviews \& evaluations, case reports and extended reports. The manuscript management system is completely online and includes a very quick and fair peerreview system, which is all easy to use. Visit http://www.dovepress.com/ testimonials.php to read real quotes from published authors. 